\title{
Negative influences of Facebook use through the lens of network analysis
}

Lien Faelens $^{1 *}$, Kristof Hoorelbeke ${ }^{1}$, Eiko Fried ${ }^{2}$, Rudi De Raedt ${ }^{1}, \&$ Ernst H.W. Koster ${ }^{1}$

1. Department of Experimental Clinical and Health Psychology, Ghent University, Henri Dunantlaan 2, B-9000, Ghent, Belgium

2. Department of Psychology, Leiden University, Wassenaarseweg 52, 2333 Leiden, The Netherlands

Please cite as follows:

Faelens, L., Hoorelbeke, K., Fried, E., De Raedt, R., \& Koster, E. H. W. (2019). Negative influences of Facebook use through the lens of network analysis. Computers in Human Behavior, 96, 13-22.

1* Corresponding author at: Department of Experimental Clinical and Health Psychology, Ghent University, Henri Dunantlaan 2, B-9000, Ghent, Belgium. Phone: +32 (0)9 26494 14; Fax: +32 (0)9 2646489

E-mail address: lien.faelens@ugent.be 


\begin{abstract}
Various recent studies suggest a negative association between Facebook use and mental health. Yet, empirical evidence for this association is mixed, raising the question under which conditions Facebook use is related to negative outcomes, such as decreased well-being. Our study addresses this question by investigating the relationship between Facebook use, rumination, depressive, anxiety-, and stress-related symptoms, taking into account potential key variables such as social comparison, contingent self-esteem, and global self-esteem. In a first study, we explored the unique relations between these constructs using state-of-the-art network analysis. Subsequently, we conducted a preregistered replication study. In both studies, social comparison and self-esteem held a central position in the network, connecting social media use with indicators of psychopathology. These findings highlight the prominent role of social comparison and self-esteem in the context of social media use and well-being. Longitudinal and experimental studies will be required to further investigate these relationships.
\end{abstract}

Keywords: Social media, Self-esteem, Depression, Anxiety, Stress

\title{
Abbreviations
}

- $\mathrm{COM-F}=$ Comparison Orientation Measure-Facebook

- $\mathrm{CSE}=$ contingent self-esteem

- $\mathrm{CSS}=$ Contingent Self-Esteem Scale

- $\quad \mathrm{FBI}=$ Facebook Intensity Scale

- $\mathrm{MSFU}=$ Multidimensional Scale of Facebook Use

- $\quad$ RRS $=$ Ruminative Responses Scale

- $\quad$ RSES = Rosenberg Self-Esteem Scale

- $\quad$ SNS $=$ Social networking sites

- DASS = Depression, Anxiety and Stress Scales 


\section{Introduction}

Social networking sites (SNS) are an important part of everyday life. Data suggests that users spend approximately 135 minutes a day on social media platforms (Statista, 2017). With 2.13 billion visitors per month, Facebook is currently the most widespread SNS, followed by Twitter and LinkedIn (Facebook, 2018). This differs considerably between different age groups, with younger people spending more time on Facebook (Ozimek \& Bierhoff, 2016). Given the importance of this SNS, researchers have become increasingly interested in possible negative consequences of Facebook use on mental well-being and the psychological constructs driving this association.

Over the years, Facebook use has been related to decreased mental well-being, as shown by elevated depressive, anxiety or (di)stress symptoms (Chen \& Lee, 2013; Shaw, Timpano, Tran, \& Joormann, 2015; Steers, Wickham, \& Acitelli, 2014). However, this relation is not supported in all studies, and sometimes the opposite pattern has been observed (for a review see Steers, 2016; Verduyn, Ybarra, Résibois, Jonides, \& Kross, 2017). For instance, research has shown that social capital may be an important factor in the positive relationship between Facebook use and well-being (e.g., Verduyn et al., 2017). Moreover, recent studies have explored the factors that may play a role in the negative association between those constructs (e.g., Appel et al., 2015; Verduyn et al., 2015; Verduyn et al., 2017) However, until now, it remains unclear under which conditions Facebook use is related to decreased mental well-being and risk for affective disorders. Given the massive use of Facebook and potential consequences in terms of depressive, anxiety or (di)stress symptoms, investigating the underlying mechanisms and processes seems crucial in understanding when Facebook use is linked to negative outcomes and vice versa. Therefore, we will first discuss the different components of 
Facebook use and introduce key variables that may link these components to risk for affective disorders: social comparison and self-esteem.

\subsection{Emotional investment and types of Facebook use}

Previous research has shown that people who experience a higher emotional connection to Facebook and other social media channels feel upset and disconnected when they are unable to access the social network site (e.g., Woods \& Scott, 2016). That is, social media platforms are often used to maintain a sense of connection (Wilson \& Gosling, 2016). As a result, when access to social network sites is restricted, users typically report more psychopathology, as indicated by elevated levels of depressive symptomatology, anxiety, and stress (Beyens, Frison, \& Eggermont, 2016; Rosen et al., 2013; Skierkowski \& Wood, 2012). In addition, previous studies suggest this is related to poorer sleep quality, a stronger orientation towards their own physical appearance, and lower levels of self-esteem, possibly due to exposure to upward comparison information (cfr. infra; Rutledge, Gillmor, Gillen, 2013; Woods \& Scott, 2016). Interestingly, in addition to people's emotional investment in social network sites, the way Facebook is used also seems to impact well-being. Prior research shows that we can make a distinction between 'active' and 'passive' Facebook use. Active Facebook use refers to "activities that facilitate interaction between the user and other Facebook friends" (Frison \& Eggermont, 2015b, p. 4). These activities, which focus on sharing new information, are mainly linked with positive mental health outcomes such as social capital and social connectedness (Verduyn et al., 2017). However, there seems to be a differential impact of active private (e.g., using Facebook Messenger) and active public Facebook use (e.g., updating Facebook status, sharing/uploading pictures; Frison \& Eggermont, 2015b). While the positive impact of active private Facebook use has been consistently shown across studies, the impact of active public Facebook use remains inconclusive (e.g., Frison \& Eggermont, 2015a). Furthermore, Passive Facebook use 
can be conceptualized as "the monitoring of other people's lives by viewing the content of others' profiles without direct exchanges between the users" (Frison \& Eggermont, 2015b, p. 4). Thus, Passive Facebook users consume content without engaging in direct exchanges with their connections. The current literature suggests that Facebook is predominantly used in a more passive way, which may elicit social comparison and increase anxiety and depressive symptoms (Appel et al., 2015; Shaw et al., 2015; Verduyn et al., 2017).

\subsection{Social comparison on Facebook}

According to Social Comparison Theory (Festinger, 1954), social comparison is a fundamental process where people tend to relate presented information to themselves for purposes of self-evaluation. Social comparison can take place in two directions: upward and downward comparison. Downward comparison occurs when comparing oneself with others who are worse off. On the other hand, upward comparison refers to selecting comparison targets who are better off (Collins, 1996). These comparisons may occur more often, especially for people who passively consume new developments in their friends' life. After all, Facebook users have the tendency to highlight positive characteristics and life events, creating a more favourable impression to others (e.g., Zhao et al., 2008). Indeed, exposure to life events and

pictures of other people's presumed flawless lives may lead to the impression that others are better and happier, which may induce feelings of inferiority. Therefore, social comparison may have a deleterious impact on people's self-evaluations and self-esteem, which in turn may contribute to the development of depressive- or anxiety symptoms (Appel, Crusius, \& Gerlach, 2015; Chou \& Edge, 2012; Orth, Robins, Trzesniewski, Maes, \& Schmitt, 2009; Wouters et al., 2013). Furthermore, online social comparison will likely have a stronger impact on individuals with (elevated risk for) affective disorders, given that this population is characterized by distortions at the level of self-esteem and social comparison (Appel et al., 2015). As such, a more thorough examination of the role of social comparison and self-esteem is warranted in 
order to gain a better understanding of the previously observed link between Facebook use and mental well-being.

\subsection{Self-esteem and Facebook}

Global self-esteem refers to the general attitude that a person has towards him- or herself. Individuals with high self-esteem report higher relationship- and job satisfaction, and

perform better on several physical and mental health outcome measures (Orth, Robins \& Widaman, 2012). Previous research has identified low self-esteem as an important risk factor for the development of affective disorders (Orth, Robins, Trzesniewski, Maes, \& Schmitt, 2009, Orth et al., 2012; Sowislo \& Orth, 2013). Given the high time investment of those individuals on social media channels as Facebook, researchers more recently started to pay special attention to the combined role of social comparison and self-esteem in relationship to negative mental health outcomes of Facebook use (e.g. Zuo, 2015). For example, research has shown that exposure to a profile of a popular or health-focused Facebook member (upward comparison) leads to a decrease in self-esteem and poorer self-evaluations (Vogel et al., 2014). This effect seems to be stronger for people characterized by low self-esteem and high depressive symptomatology (e.g., Appel et al., 2015). In line with these findings, Wang, Wang, Haskin, and Hawk (2017) showed that both upward comparison and self-esteem mediated the relationship between passive social network usage and subjective well-being.

Although the current literature focused on level of self-esteem, self-esteem can be conceptualized as both a stable trait and more dynamic state that fluctuates during the day in response to daily stressors and boosts. Therefore, another variable that may mediate the relationship between Facebook use and indicators of psychopathology is contingent self-esteem (CSE), which is also an important predictor of depressive symptomatology (Wouters et al., 2013). CSE can be defined as the degree to which one's self-esteem depends on matching certain standards of social standing, physical appearance, good grades, etc. (Deci \& Ryan, 
1995). Based on the extant literature on self-esteem we propose that especially the construct CSE will be important in the context of social media use, since people who show high CSE are more sensitive to the information and feedback provided on Facebook (Kernis, 2003; Pettijohn, LaPiene, Pettijohn, \& Horting, 2012; Stefanone, Lackaff, \& Rosen, 2011). We hypothesize that people with a high CSE will use information derived from Facebook to determine to what extent they have been successful in reaching their own personal standards. This can lead to self-esteem boosts when they meet their personal standards, but may also lead to the opposite if standards are not reached. For example, if personal self-worth is highly dependent on social standing, receiving a high amount of likes on a new status update or profile picture will lead to positive self-evaluations. Over time, these fluctuations in self-esteem - together with rumination - could cascade into negative emotional experiences such as stress-, anxiety-, and depressive symptoms (Kernis, 2003; Wouters et al., 2013).

\subsection{Current Study}

Network methodology gained traction in the last few years in psychological research and allows to gain insight in the complex relationships between variables or constructs in a data-driven manner. Given that our literature review demonstrates that these constructs are highly interrelated, with a high potential for bidirectional relationships, we will make use of psychological network models (Costantini et al., 2015; Fried et al., 2018; Hoorelbeke, Marchetti, De Schryver, \& Koster, 2016). In this way, we investigated how key psychological factors such as social comparison, contingent self-esteem, global self-esteem, and rumination are involved in the relationship between Facebook use and affect. Based on the prior literature, we expected to find the following relationships:

(H1) (Passive) Facebook use and Facebook intensity will be related to social comparison behaviour; 
(H2) Social comparison will link (intensity) of Facebook use with self-esteem;

(H3) Self-esteem will link rumination, anxiety-, depressive-, and stress related symptoms with social comparison and (intensity of) Facebook use.

\section{Study 1 - Method}

\subsection{Participants}

Two hundred and seven participants recruited through the online crowdsourcing platform Prolific Academic (http://prolific.ac) completed self-report questionnaires regarding their Facebook use and mental well-being. Participation was restricted to respondents between 18 and 35 years who had a Facebook profile. Sample characteristics are reported in the supplemental material. All participants provided informed consent prior to completing the survey and received financial reimbursement for their participation. This study was approved by the local institutional review board.

\subsection{Measures}

Facebook use. The Multidimensional Scale of Facebook Use (MSFU; Frison \& Eggermont, 2015) is a 10-item self-report measure. Using a 7-point Likert scale, which ranged from 1 ("never"”) to 7 ("several times per day"), participants have to rate the extent to which they practiced 10 Facebook activities. This instrument measures three types of Facebook activities: passive Facebook use (e.g., "How often do you visit a Facebook profile of a Facebook friend?”), active private Facebook use (e.g., "How often do you chat with someone on Facebook?"), and active public Facebook use (e.g., "How often do you post a picture or video on your own Facebook timeline?"). However, we decided to exclude one item of the passive Facebook use subscale (“How often do you read your news feed?") because this item loaded highly on another subscale (i.e., active private Facebook use). This decision is in line with previous research 
(Frison \& Eggermont, 2015) (see Table 3 in the supplemental material for details of the PCA). The internal consistency of the three subscales in the current study was as follows: passive Facebook use $(\alpha=.89)$, active private Facebook use $(\alpha=.89)$, and active public Facebook use $(\alpha=.94)$.

Facebook intensity. The Facebook Intensity Scale (FBI; Ellison, Steinfield, \& Lampe, 2007) is designed to measure people's emotional connection with Facebook activities $(\alpha=.88)$. In this study, we used the six attitudinal items, rated on a 5-point Likert scale, measuring participants' connectedness to this SNS (e.g., "I am proud to tell people I'm on Facebook").

Social comparison on Facebook. Social comparison on Facebook was assessed using the Comparison Orientation Measure-Facebook (COM-F; Steers et al., 2014). The COM-F is an adaptation of the widely used Iowa-Netherlands Comparison Orientation Measure (INCOM; Gibbons \& Buunk, 1999), where the items were modified to fit a Facebook context. The COMF comprises 11 items that were rated on a 5-point likert scale (e.g., "When I am on Facebook, I compare myself with others with respect to what I have accomplished in life") and shows good reliability $(\alpha=.91)$.

Contingent self-esteem. The Contingent Self-Esteem Scale (CSS; Paradise \& Kernis, 1999) consists of 15 items each rated on a 5-point likert scale from 1 ("not at all like me") to 5 ("very much like me"). It assesses self-esteem contingencies with regard to issues as gaining approval of peers and meeting personal standards (e.g., "My overall feelings about myself are heavily influenced by what I believe other people are saying or thinking about me") and has a good reliability $(\alpha=.85)$.

Global self-esteem. The Rosenberg Self-Esteem Scale (RSES; Rosenberg, 1965) is a wellestablished instrument that assesses global feelings of self-worth or self-acceptance $(\alpha=.92)$. The 10-item instrument instructs participants to rate whether they strongly agree, agree, 
disagree, or strongly disagree with the presented statements (e.g., "On the whole, I am satisfied with myself').

Rumination. The Ruminative Responses Scale (RRS; Treynor, Gonzalez, \& Nolen-Hoeksema, 2003) is a measure for trait rumination and indicates how often participants generally engage in repetitive negative thinking $(\alpha=.94)$. This scale contains 22 items, rated on a 4-point Likert scale from 1 ("almost never") to 4 (“almost always") (e.g., "think about how alone you feel”).

Negative emotional states of depression, anxiety, and stress. The Depression, Anxiety and Stress Scales (DASS-21; Lovibond \& Lovibond, 1995) is a screening measure of distress that contains three subscales representing the extent to which participants report experiencing depressive- (e.g., "I felt that life was meaningless", $\alpha=.93$ ), anxiety- (e.g., "I felt I was close to panic", $\alpha=.86$ ), and stress symptoms (e.g., "I found myself getting agitated", $\alpha=.88$ ). Each scale is represented by 7 items with response options ranging from 0 ("Did not apply to me at all”) to 3 ("Applied to me very much, or most of the time").

\subsection{Data analysis}

Data analysis was conducted in $\mathrm{R}$ version 3.3 .2 (see supplemental material for the version info of all $\mathrm{R}$ packages we used for the analyses). After inspection of the data and identifying some skew, we used the nonparanormal transformation via the huge package (Zhao et al., 2015) to improve normality. We then proceeded with network analysis. This methodology enables the visualisation of reciprocal relations and feedback loops, without making strong assumptions about directionality.

Although these models are mathematically equivalent to structural equation models, at least under certain conditions (Kruis \& Maris, 2016), the theories that motivate using these models differ from each other. Depression symptoms, for instance, are correlated, and there are at least two principled explanations for these correlations. The first is that depression is a brain 
disorder, which causes the observable symptoms, making them correlated. This would imply that a common cause generated the data, in which we should fit a unidimensional factor model that accounts for measurement error. Fitting a network model would make little sense. An alternative theory is that correlations stem from causal interactions and feedback loops among symptoms (Borsboom 2017), which would motivate the use of a network model. In this case, i.e. when the data generating mechanism is not a factor model, fitting a factor model can also lead to severe bias in the parameter estimates (Rhemtulla, van Bork, Borsboom, 2018). Because we were interested in mutual interactions among a host of different items, we estimated a Gaussian Graphical Model for our cross-sectional data, and did not include SEM analyses in the paper We estimated the Gaussian Graphical Model (also called regularized partial correlation network) using the qgraph package (Epskamp, Cramer, Waldorp, Schmittmann, \& Borsboom, 2012). Partial correlations provide an estimate of the unique shared variance of every node to other nodes in the model. The Gaussian Graphical Model uses regularization to obtain a sparse network in which spurious edges are removed (Epskamp \& Fried, 2018). For this purpose, we relied on the Graphical Least Absolute Shrinkage and Selection Operator (gLASSO; Friedman, Hastie, \& Tibshirani) with Extended Bayesian Information Criterion model selection (EBIC; $\gamma=0.5)$.

The potential importance of nodes within a given network can be gauged by investigating so-called centrality indexes. For this purpose, strength, closeness, and betweenness centrality are commonly estimated. Node strength is calculated as the sum of absolute edge weights connected to each node, whereas betweenness refers to the amount of times that a given node lies on the shortest path between two other nodes. Closeness represents the inverse of the sum of distances from a given node to all other nodes (Costantini et al., 2015; Epskamp \& Fried, 2018; Opsahl, Agneessens, \& Skvoretz, 2010). In addition, we estimated node predictability, referring to the variance of each node explained by its neighbors ( Haslbeck 
\& Fried, 2017), using the $m g m$ package (Haslbeck \& Waldorp, 2015). Following the network estimation, we assessed accuracy and stability of the network using the bootnet package (Epskamp \& Fried, 2015). In line with the procedure described by Epskamp, Borsboom, and Fried (2018), we used bootstrapping to estimate the accuracy of the given network by investigating the sampling variability in edge weights, and plotting significant differences between edge weights. Furthermore, stability of the centrality indices was assessed using a casedropping subset bootstrap, representing the extent to which the order of centrality indices remain stable within subsets of the data. In order for centrality indices to be considered stable, Epskamp et al. (2018) suggest the resulting correlation stability coefficient should not be $<0.25$ and preferably $\geq 0.50$. Networks were plotted using a modification of the FruchtermanReingold's algorithm (Fruchterman \& Reingold, 1991), resulting in a network in which more strongly connected nodes hold a central position. In addition, strength of the connections is reflected by thickness of the edges whereas valence of the associations is depicted by edge color. In particular, blue represents unique positive associations between two nodes whereas red represents negative associations. Predictability of a node is depicted as a pie chart in the rings around nodes within the network (e.g., Fried et al., 2018). Here, the blue area in the outer ring of nodes represents the percentage of variance of the node that is explained by all neighboring nodes ${ }^{2}$.

\section{Study 1 - Results}

$2 *$ In line with Haslbeck and Fried (2017), estimations of predictability of nodes within a network are computed based on models derived from $\mathrm{mgm}$ (which uses a node-wise regression approach to estimate the network structures), whereas the presented regularized partial correlation network models were estimated with the qgraph package (gLASSO and EBIC model selection) in line with the pre-registration which uses a different estimation approach (inversion of the covariance matrix). As a result, the aggregated output relies on two different estimation methods (the edges on the qgraph estimation, the predictability on the mgm estimation). Importantly, the adjacency matrices obtained from both estimation methods were moderately correlated (Study 1: $r=.68$; Study 2: $r=.63$ ), suggesting that similar models are obtained using different estimation methods. 
Descriptive statistics of the variables of interest are reported in the supplemental material. The regularized partial correlation network (Figure 1) depicts two conceptual clusters of nodes, which are indirectly connected. The first cluster contains indicators of psychopathology, among which severity of depressive- ('Depression'), stress- ('Stress'), and anxiety ('Anxiety') symptoms, as well as rumination ('RRS') as a transdiagnostic risk factor for affective disorders. The second cluster, on the other hand, encompasses indicators of social media use, among which type of Facebook use ('MSFU_Private', 'MSFU_Public', 'MSFU_Passive') and intensity of Facebook use ('FBI'). Both clusters connect via social comparison ('COMF'), contingent self-esteem ('CSS') and global self-esteem ('RSES'). That is, with the exception of six small edges (edge weights ranging from -.03 to .06) directly linking anxiety symptoms to private and public Facebook use, the remainder of nodes of the two clusters are not directly connected (i.e. nodes do not share variance after partialling out all other nodes).

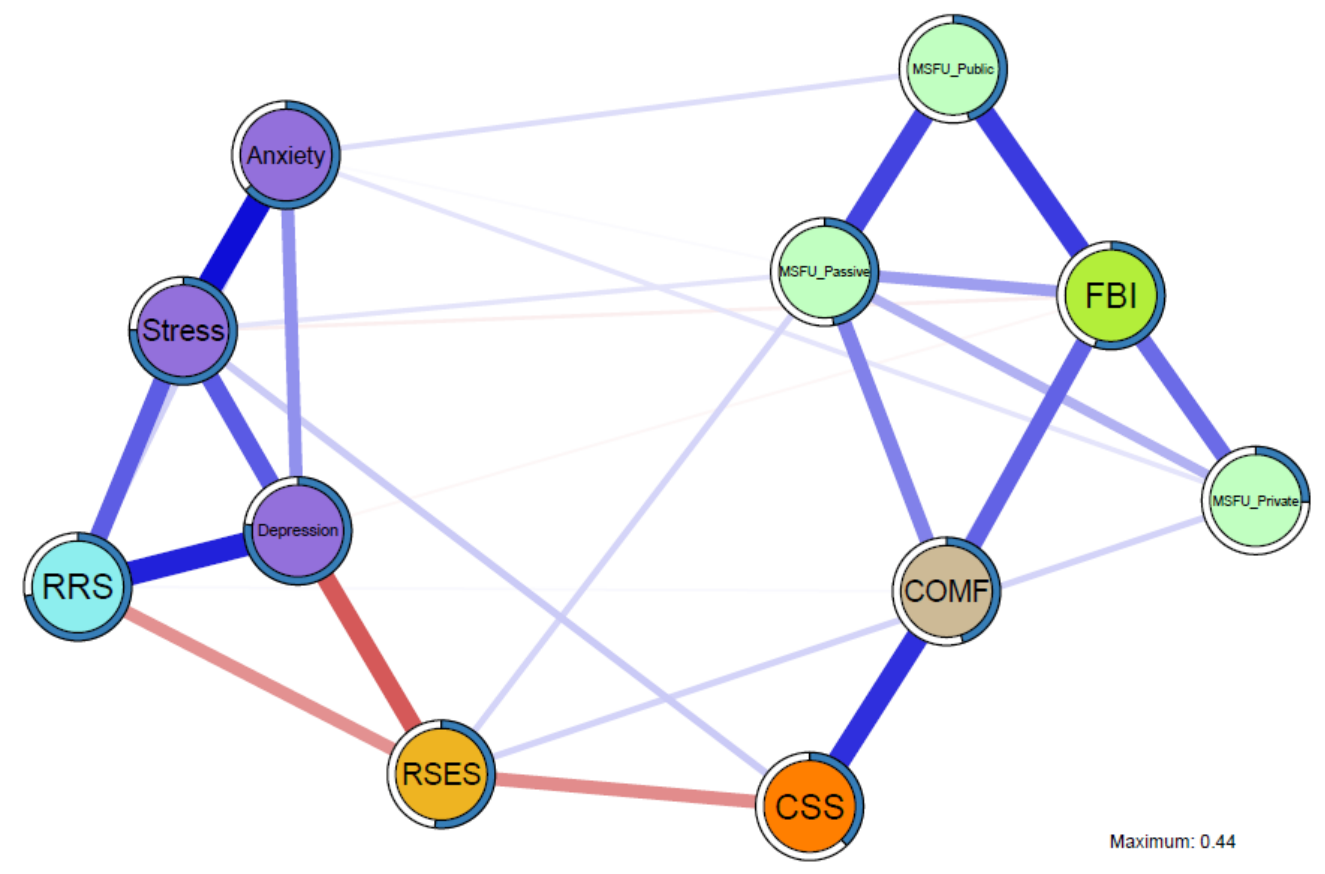

Figure 1. Regularized Partial Correlation Network Study 1 
The central role for social comparison (COMF), contingent self-esteem (CSS), and global selfesteem (RSES) as bridge constructs in the network is supported by centrality metrics Closeness and Betweenness (Figure 2). These suggest that social comparison, contingent self-esteem, and global self-esteem often lay on the shortest path between two nodes, offering the smallest distance as they connect different nodes with one another in the network model. Nodes that are part of the psychopathology or social media use cluster score highest on the Strength index. Stability analysis of the centrality indices (supplemental material) suggests good stability for Strength (0.59) and acceptable stability for Betweenness (0.29) and Closeness (0.29; see supplemental material for all other stability and accuracy analyses such as the edge weights accuracy)
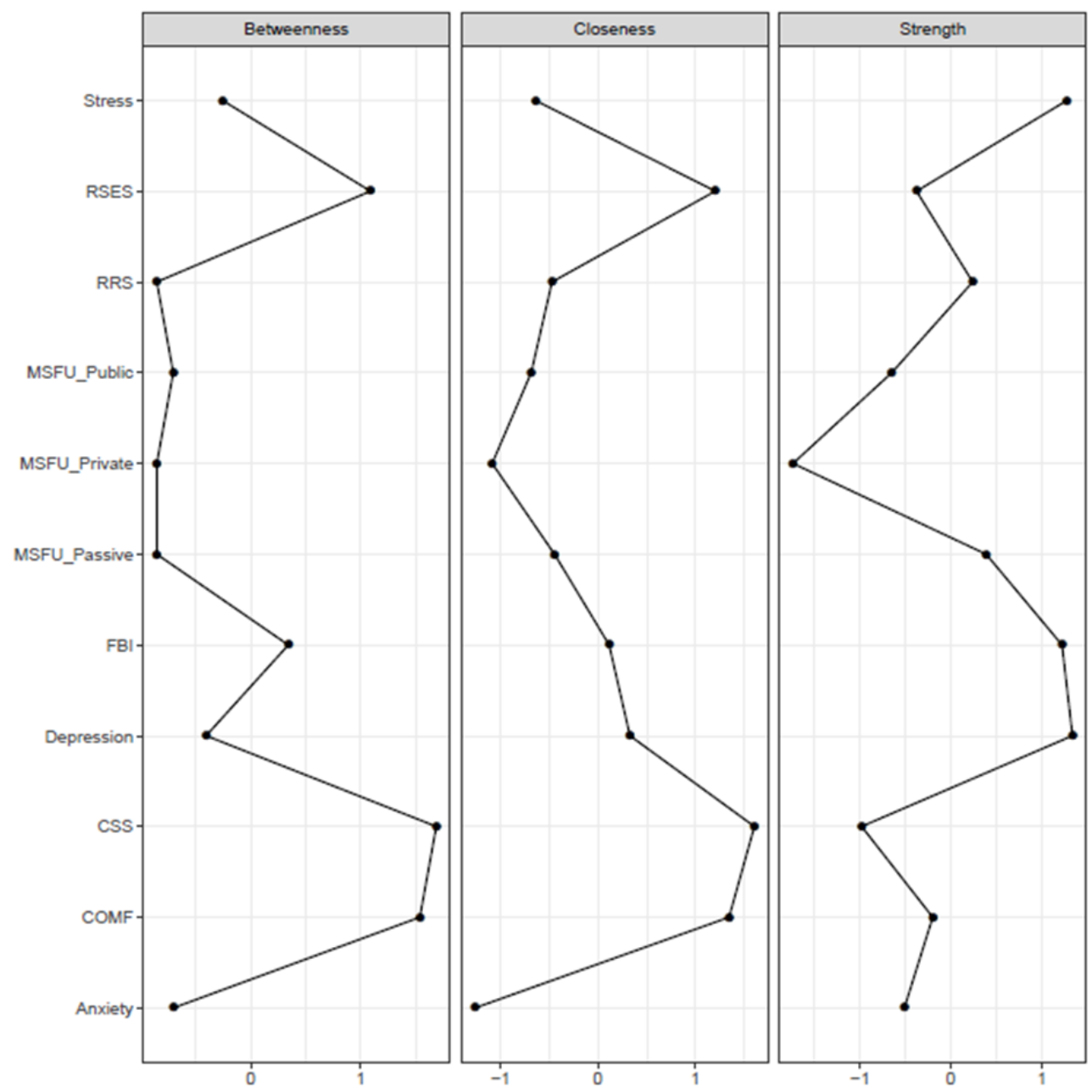

Figure 2. Standardized centrality indices Study 1 


\section{Interim conclusion}

In this first exploratory study, we found that social comparison and (contingent) self-esteem hold a central position in the network, connecting social media use with self-reported depressive, anxiety, and stress symptoms. In the second study, we tested whether this network structure could be replicated. Therefore, we pre-registered our hypotheses, design, and data analytical strategy on Open Science Framework (osf.io/ahgxk). This platform allows researchers to document and share study designs, materials and data which facilitates open collaboration.

\section{Study 2 - Method}

\subsection{Power Analysis}

We aimed to recruit 500 participants, as pre-registered on Open Science Framework (osf.io/ahgxk). This number was based on a simulation study. Because many parameters are estimated in network models, power analysis has remained an unexplored topic thus far. Only recently, a novel methodology was developed, tested, validated, and implemented in the Rpackage bootnet via the function netSimulator (Epskamp \& Fried, 2018).

To the best of our knowledge, the present work uses for the first time power analysis to establish an optimal sample size for a registered confirmatory study with network analysis. In detail, we simulated datasets with different sample sizes based on the parameters of the network model obtained from Study 1. We then used three indices to determine the sample size required to reliably discover this true network structure in the simulated datasets: correlation (how strongly is the estimated network correlated to the true network), sensitivity (how well does the estimated network discover edges), and specificity (how well does the estimated network discover absent edges). We also investigated how well centrality indices are retrieved. 
The results of the simulation study suggested to collect data of at least 450 participants. That is, at $N=450$ correlation and sensitivity reached values of $\geq .90$, indicators for centrality reached values of about .80 , and specificity reached .70 . As expected, specificity did not benefit from further increasing sample size (.70 is a comparably high sensitivity value for these types of network models, since the regularization techniques employed sacrifice specificity to maximize sensitivity (Epskamp \& Fried, 2018). Similarly, centrality indices only show limited benefits from further increasing the sample size (see supplemental material). As a result, taking into account potential loss of participants due to incorrect responses on the check items (cf. estimated at $10 \%$ ), we aimed to recruit 500 participants in order to reach $N=450$, as preregistered on Open Science Framework (osf.io/ahgxk).

\subsection{Participants}

We recruited 500 participants aged 18-35 via Prolific Academic. However, seven participants completed the survey without entering a completion code. As such, data was collected for a total of 507 participants. Based on two reading-check questions, we had to exclude 39 participants. This led to a final sample of 468 participants, well above the pre-specified threshold (for sample characteristics, see supplemental material). Participants provided informed consent prior to completing the survey and received financial compensation for participation.

\subsection{Measures}

The survey included the same questionnaires as Study 1, except that we now added two reading-check items (e.g., "As a reading check could you please select the answer 'I disagree strongly'"'). 


\subsection{Data availability}

The datasets and data analysis scripts generated during and/or analysed during the current study will be shared open access on https://osf.io/ahgxk/ following peer review of the manuscript.

\subsection{Data analysis}

Data analysis followed the same procedure as described in Study 1. To evaluate the extent to which the network structure obtained in the first dataset replicates in the second dataset, we performed two sets of analyses. First, we determined the similarity of the two results, by correlating the two adjacency matrices (i.e., the set of regularized partial correlations that is visualized in the graphs), the centrality indices, and the predictability of nodes. Second, to determine the differences of the two results, we used permutation tests for network structure invariance and connectivity invariance using the R-package NetworkComparisonTest (van Borkulo, Epskamp, \& Millner, 2016; van Borkulo et al., Submitted). Network structure, as the name suggests, determines whether the two graphs differ from each other statistically, whereas connectivity invariance (or global strength invariance) tests whether the sum of all absolute edges across the two networks differs. In order to allow for visual comparison between the network models obtained from both studies, we constrained the layout of the networks and the maximum edge values to be equal when plotting the two networks (Figure 3).

\section{Study 2 - Results}

Descriptive statistics of all variables are reported in the supplemental material. Figure 3 presents the network models obtained from Study 1 and 2. For the unconstrained layout of the two networks, and all model output, see supplemental material. 
In line with our expectations, both models show strong overlap as seen by: (a) comparison of the adjacency matrices of Study 1 and $2(r=.95)$, (b) comparison of the centrality indices (Strength: $r=.80$; Closeness: $r=.91$; Betweenness: $r=.81$; Figure 4), and (c) comparison of predictability of nodes (Study 1: mean $R^{2}=54 \%$, Study 2: mean $R^{2}=50 \% ; r=$ .98 ; see supplemental material). In addition, the Network Comparison Test yielded no significant differences in terms of network structure (test of invariant network structure; $M=$ $0.12, p=.80$ ) or overall strength of connectivity (test of invariant global strength; Study $1=$ 4.94, Study $2=4.74, S=0.20, p=.67)$. These findings suggest that the network models derived from Study 1 and Study 2 show an identical structure and a similar overall level of connectivity. In line with Study 1, stability analysis of the network model obtained in Study 2 suggests acceptable stability for Closeness (0.44) and good stability for Strength (0.75). In contrast, Betweenness, on a global level, was unstable ( 0.00 ; for detailed analyses of stability and accuracy, see supplemental material). A coefficient of 0.00 is unexpected, and we therefore performed a more detailed analysis at the level of individual nodes (item-wise case-dropping subset bootstrap), which revealed that the stability problems are due to several nodes from the Psychopathology cluster and Social media use cluster that show to be unstable in terms of obtained order of Betweenness within subsets of the data. Importantly, the order of the bridging nodes contingent self-esteem (CSS) and social comparison (COMF) remained stable within subsets of the data, and were consistently ranked highest in terms of Betweenness. This means that, while the general order of Betweenness should not be interpreted, contingent self-esteem and social comparison can be interpreted as the reliably most central nodes in the network in terms of Betweenness centrality. 

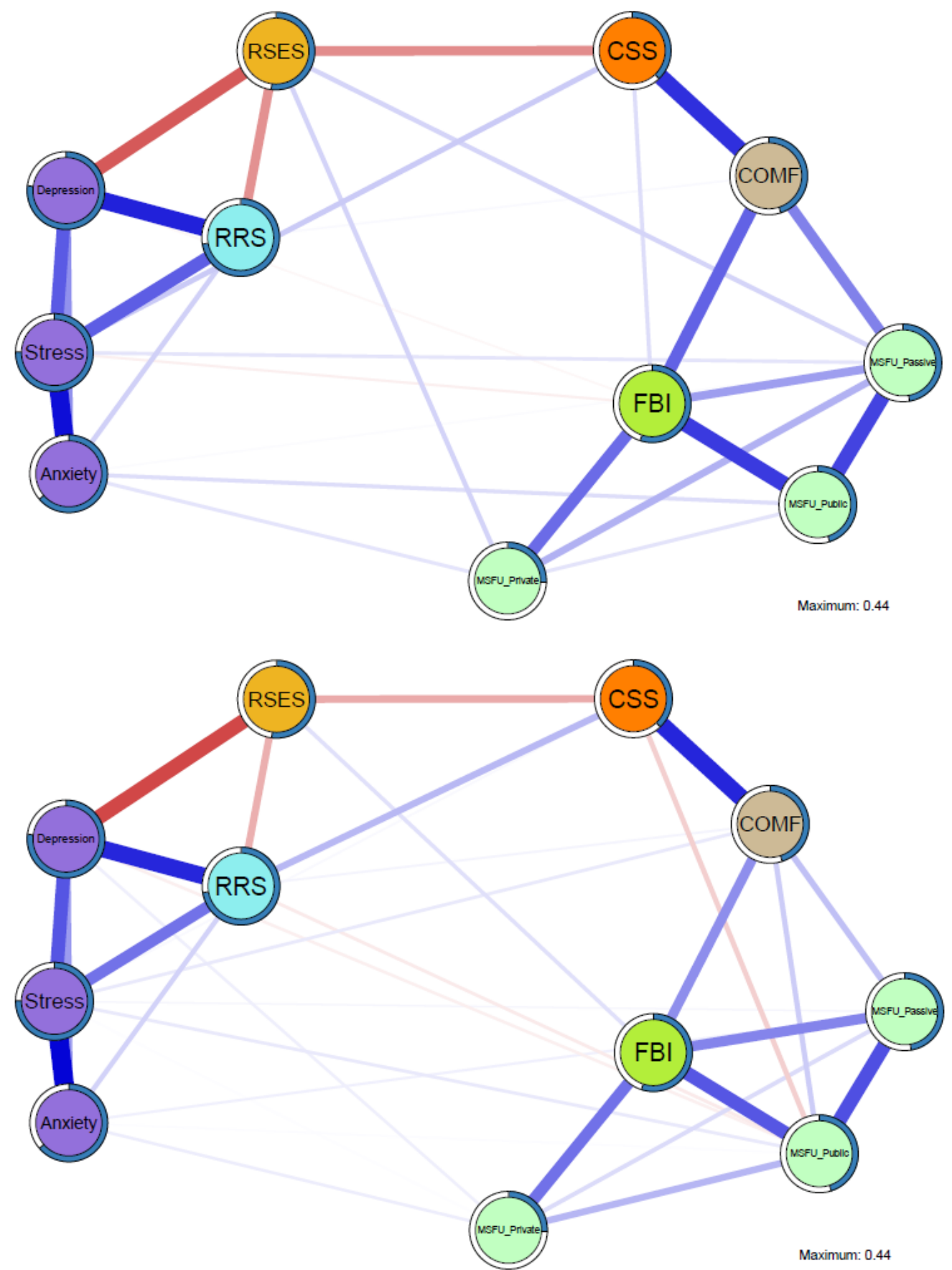

Figure 3. Comparison of regularized partial correlation networks obtained from Study 1 (upper model) and Study 2 (lower model) 

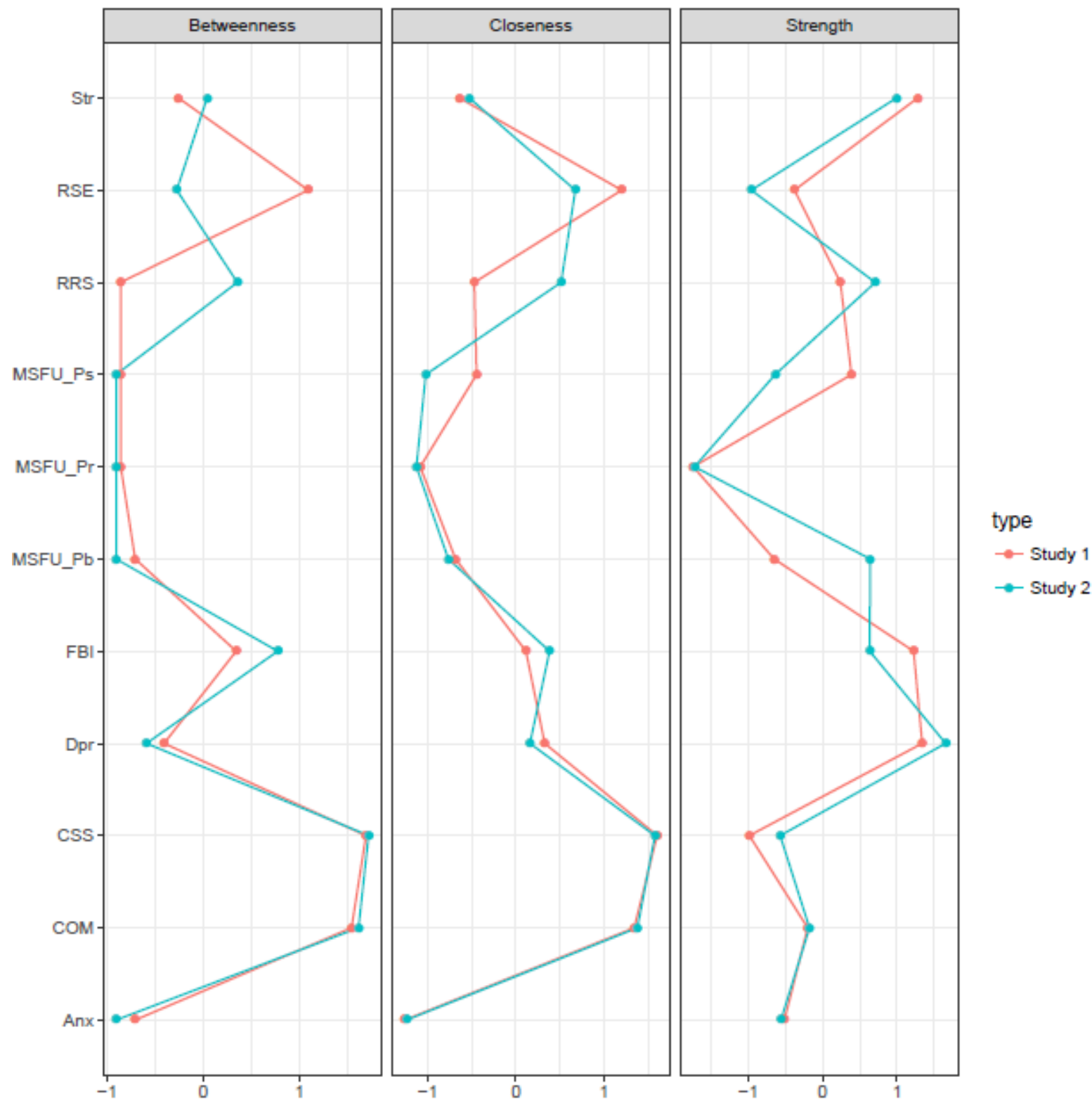

Figure 4. Standardized Centrality Indices Study 1 and Study 2 


\section{General discussion}

\subsection{Discussion of the key findings}

A large body of literature links Facebook use with detrimental outcomes such as decreases in mental well-being (Kross et al., 2013; Verduyn et al., 2017). However, the mechanisms underlying these associations need further investigation. In line with Wang et al. 2017), we hypothesized to find that indicators of (intensity of) Facebook use would be linked to reduced well-being - as shown by elevated depressive-, anxiety-, and stress-related symptoms - via social comparison and self-esteem. For this purpose, we set up an exploratory study (Study 1), modeling the unique associations between each of these constructs. In particular, we relied on network analysis as this offers a data-driven view of the complex relationships between the numerous variables of interest, modeling the predictability and unique contribution of each of the constructs in the network. Second, we conducted a preregistered confirmatory study (Study 2) to test the robustness of our network. Using a larger sample size and identical procedure, we replicated the network structure obtained in Study 1. That is, in line with our hypotheses, the partial correlation networks revealed a bridging role of social comparison and self-esteem (contingent- and global self-esteem), connecting social media use with indicators of psychopathology. More specifically, we confirmed that: (a) (Passive) Facebook use and Facebook intensity were linked to social comparison behaviour (H1), (b) Social comparison linked (intensity) of Facebook use with self-esteem (H2), and (c) Self-esteem linked rumination, anxiety-, depressive-, and stress related symptoms with social comparison and (intensity of) Facebook use (H3).

People who report higher levels of Facebook use, experience a higher emotional and stronger need to be connected. This is the case for all types of Facebook use, since they all show a strong connection with Facebook intensity, which was in turn linked to the psychopathology 
indicators via social comparison and self-esteem. This is in line with previous research findings, showing that the need to be connected is accompanied by reduced self-esteem levels and elevated depression, anxiety and stress levels (Beyens, Frison, \& Eggermont, 2016; Blachnio, Przepriorka \& Pantic, 2016; Rosen et al., 2013; Skierkowski \& Wood, 2012).

Interestingly, our results also relate to previous research findings demonstrating the detrimental influence of passive Facebook use on various outcome measures (e.g., decreased mental well-being; Verduyn et al., 2015; Verduyn et al., 2017). In line with this idea, results of both studies consistently showed that passive Facebook use was directly connected with social comparison, which linked this construct to the psychopathology indicators via self-esteem. Moreover, in our second study, we also found a direct connection between active public Facebook use and social comparison. We hypothesize that this connection was only found in the second dataset due to the larger sample size, enhancing statistical power to detect weak edges. This finding should be followed up in future work.

Although studies with multiple time points will be necessary to test the role of the bridging variables, our findings are consistent with the rapidly growing literature suggesting that social comparison might mediate the relationship between Facebook use and depressive symptomatology (Steers et al., 2014). Because Facebook profiles tend to strategically emphasize people's most desirable traits, Facebook users are constantly exposed to the positive life events and successes of others (Zhao et al., 2008). In this context, selective confrontation with success experiences of others may trigger repetitive negative thinking regarding one's imperfections, which forms a well-known risk factor for the aetiology and maintenance of affective disorders. Arguably, this effect will be stronger for depressive or anxious individuals who already report a higher tendency to ruminate (Feinstein et al., 2013; McLaughlin \& NolenHoeksema, 2010). 
Surprisingly, less research has focused on the role of self-esteem in the relationship between Facebook use and indicators of psychopathology. Although, there is emerging evidence showing that exposure to Facebook profiles of (favourable) others (e.g., attractive, popular or healthy individuals) provokes poorer self-evaluations and a lower state self-esteem (Appel et al., 2015; Vogel et al., 2014). These negative self-evaluations can in turn elicit depressive symptomatology (Orth et al., 2009). To our knowledge, only two studies demonstrated the importance of the construct contingent self-esteem in relation to Facebook use (Pettijohn et al., 2012; Stefanone et al., 2011). However, these studies only mapped the association between (intensity of) Facebook use and contingent self-esteem and did not include any measures of psychopathology. Therefore, the current studies serve as an initial step in relating Facebook use, (contingent) self-esteem, and well-being. Moreover, we replicated the network structure obtained in Study 1, suggesting a central role of social comparison and contingent self-esteem. Especially in a social media context, these seem important constructs to take into account. That is, users are constantly exposed to interpersonal feedback (likes/comments) and self-promoting information of their Facebook friends. This may elicit self-esteem fluctuations and increase the risk for developing depressive symptomatology (Kernis, 2003; Wouters et al., 2013).

\subsection{Limitations}

The current study has several limitations. First, the cross-sectional nature of the study does not permit causal conclusions ${ }^{3}$. This means that (intensity of) Facebook use can lead to reduced well-being - as shown by elevated depressive-, anxiety-, and stress-related symptoms - via social comparison and self-esteem. However, that the reverse pattern is also plausible

\footnotetext{
${ }^{3}$ In a cross-sectional dataset edge A-B can exist because $\mathrm{A}->\mathrm{B}, \mathrm{A}<-\mathrm{B}$ or $\mathrm{A}<->\mathrm{B}$
} 
considering the fact that people with (an elevated risk for) affective disorders show distortions at the level of self-esteem and social comparison processes, which may impact their Facebook use (Appel et al., 2015). For example, research suggests that higher anxiety and/or depression levels are accompanied by higher levels of passive Facebook use (Escobar-Viera et al., 2018; Shaw et al., 2015). However, it does allow generating hypotheses about the causal structure in the data which can be tested in future longitudinal and experimental research. Second, we included eleven nodes based on previous research findings about Facebook use and risk for affective disorders. Given that we mainly focused on possible mediators that were likely to undermine well-being we might be overlooking important constructs, which could play an important role in the relationship between Facebook use and reduced well-being. However, the high average predictability across both studies suggests that, although some variables are missing, we capture important theoretical constructs in the context of social media and psychopathological processes. Future studies could also include constructs as 'social capital' and 'social connectedness', which might be important variables in the positive relationship between Facebook use and well-being (Verduyn et al., 2017). Finally, the Network Comparison Test might not have been sufficiently powered to detect differences between both models. However, given the small test statistics, potential differences are likely to have been small. In line with this, our findings suggest that the weight matrices, centrality metrics, and explained variance of nodes obtained from Study 1 and Study 2 strongly overlap.

\subsection{Implications for future research}

Our study provides key insights regarding how different psychological factors are involved in the association between Facebook use and risk for affective disorders. The next step for research will be to elucidate the dynamic effects of Facebook use on well-being. Therefore, future studies should use experience-sampling methods to assess Facebook use, social comparison, self-esteem and indicators of psychopathology throughout the day to 
clarify the temporal nature of the associations. Provided that SNS as Facebook play such a key role in the social life of adolescents and young adults, improving our understanding of the interactions online and their relation to psychological constructs is of crucial importance.

\section{Conclusion}

Social network sites such as Facebook are immensely popular and have become an important part of life. Yet it remains unclear when and under which conditions they may be linked to depressive-, anxiety-, and stress symptoms. A substantial amount of research suggests the importance of social comparison in this relationship, but the role of self-esteem needed further investigation. Therefore, the presented studies serve as an initial step in relating Facebook use with social comparison, (contingent) self-esteem, and indicators of psychopathology. In a first exploratory study, we deployed network modelling to look into the complex relationships between our variables of interest. Based on our initial findings, we conducted a power analysis and set-up a replication study which we preregistered (Study 2). Both studies point out the central role of social comparison and self-esteem, increasing our understanding of the association between Facebook use and psychopathology. 


\section{References}

Appel, H., Crusius, J., \& Gerlach, A. L. (2015). Social comparison, envy, and depression on Facebook: A study looking at the effects of high comparison standards on depressed individuals. Journal of Social and Clinical Psychology, 34(4), 277-289. doi:10.1521/jscp.2015.34.4.277

Beyens, I., Frison, E., \& Eggermont, S. (2016). “I don’t want to miss a thing”: Adolescents' fear of missing out and its relationship to adolescents' social needs, Facebook use, and Facebook related stress. Computers in Human Behavior, 64, 1-8. doi:10.1016/j.chb.2016.05.083

Błachnio, A., Przepiorka, A., \& Pantic, I. (2016). Association between Facebook addiction, self-esteem and life satisfaction: A cross-sectional study. Computers in Human Behavior, 55, 701-705. doi:10.1016/j.chb.2015.10.026

Borsboom, D. (2017). A network theory of mental disorders. World Psychiatry, 16(1), 5-13. doi:10.1002/wps.20375

Chen, W., \& Lee, K. H. (2013). Sharing, liking, commenting, and distressed? The pathway between Facebook interaction and psychological distress. Cyberpsychology, Behavior, and Social Networking, 16(10), 728-734. doi:10.1089/cyber.2012.0272

Chou, H. T. G., \& Edge, N. (2012). “They are happier and having better lives than I am": The impact of using Facebook on perceptions of others' lives. Cyberpsychology, Behavior, and Social Networking, 15(2), 117-121. doi: 10.1089/cyber.2011.0324

Collins, R. L. (1996). For better or worse: The impact of upward social comparison on selfevaluations. Psychological Bulletin, 119(1), 51-69. doi:10.1037/0033-2909.119.1.51

Costantini, G., Epskamp, S., Borsboom, D., Perugini, M., Mottus, R., Waldorp, L. J., \& Cramer, A. O. J. (2015). State of the aRt personality research: A tutorial on network analysis of 
personality data in R. Journal of Research in Personality, 54, 13-29. doi:10.1016/j.jrp.2014.07.003

Deci, E., \& Ryan, R. (1995). Human autonomy. In M. Kernis (Ed.), Efficacy, Agency, and Self Esteem SE - 3 (pp. 31-49). doi: 10.1007/978-1-4899-1280-0_3

Escobar-Viera, C. G., Shensa, A., Bowman, N. D., Sidani, J. E., Knight, J., James, A. E., \& Primack, B. A. (2018). Passive and Active Social Media Use and Depressive Symptoms Among United States Adults. Cyberpsychology, Behavior, and Social Networking, 21(7), 437-443. doi:10.1089/cyber.2017.0668

Ellison, N. B., Steinfield, C., \& Lampe, C. (2007). The Benefits of Facebook "Friends": Social Capital and College Students' Use of Online Social Network Sites. Journal of Computer-Mediated Communication, 12(4), 1143-1168. doi:10.1111/j.10836101.2007.00367.x

Epskamp, S., Borsboom, D., \& Fried, E. I. (2018). Estimating psychological networks and their accuracy: A tutorial paper. Behavior Research Methods, 50, 195-212. doi: $10.3758 / \mathrm{s} 13428-017-0862-1$

Epskamp, S., Cramer, A., Waldorp, L., Schmittmann, V. D., \& Borsboom, D. (2012). qgraph: Network visualizations of relationships in psychometric data. Journal of Statistical Software, 48, 1-18. doi:10.18637/jss.v048.i04

Epskamp, S., \& Fried, E. I. (2018). A Tutorial on Regularized Partial Correlation Networks. Psychological Methods. 23(4), 617-634. doi: 10.1037/met0000167

Epskamp, S., \& Fried, E. I. (2017). bootnet: Bootstrap Methods for Various Network Estimation Routines. R package version 1.0.1

Facebook (2018). Facebook Reports Fourth Quarter and Full Year 2017 Results. Retrieved from https://investor.fb.com/investor-news/press-release-details/2018/FacebookReports-Fourth-Quarter-and-Full-Year-2017-Results/default.aspx 
Feinstein, B. A., Hershenberg, R., Bhatia, V., Latack, J. A., Meuwly, N., \& Davila, J. (2013). Negative social comparison on Facebook and depressive symptoms: Rumination as a mechanism. Psychology of Popular Media Culture, 2(3), 161-170. doi:10.1037/a0033111

Festinger, L. (1954). A theory of social comparison processes. Human relations, 7(2), 117-140.

Fried, E. I., Eidhof, M. B., Palic, S., Costantini, G., Huisman-van Dijk, H. M., Bockting, C. L. H., . . . Karstoft, K-I. (2018). Replicability and generalizability of PTSD networks: A cross-cultural multisite study of PTSD symptoms in four trauma patient samples. Clinical Psychological Science. doi: 10.17605/OSF.IO/2T7QP

Friedman, J. H., Hastie, T., \& Tibshirani, R. (2014). glasso: Graphical lasso- estimation of Gaussian graphical models. R package version 1.8.

Frison, E., \& Eggermont, S. (2015a). Exploring the Relationships Between Different Types of Facebook Use, Perceived Online Social Support, and Adolescents' Depressed Mood. Social Science Computer Review, 34(2), 153-171. doi:10.1177/0894439314567449

Frison, E., \& Eggermont, S. (2015b). Toward an Integrated and Differential Approach to the Relationships Between Loneliness, Different Types of Facebook Use, and Adolescents' Depressed Mood. Communication Research, 1-28. doi:10.1177/0093650215617506

Fruchterman, T., \& Reingold, E. (1991). Graph drawing by force-directed placement. Software: Practice and Experience, 21, 1129-1164. doi:10.1002/spe.4380211102

Gibbons, F. X., \& Buunk, B. P. (1999). Individual differences in social comparison: Development of a scale of social comparison orientation. Journal of Personality and Social Psychology, 76(1), 129-142. doi:10.1037/0022-3514.76.1.129

Haslbeck, J. M. B., \& Fried, E. I. (2017). How predictable are symptoms in psychopathological networks? A reanalysis of 18 published datasets. Psychological Medicine, 47, 27672776. doi: 10.1017/S0033291717001258 
Haslbeck, J. M. B., \& Waldorp, L. J. (2015). mgm: Estimating Time-Varying Mixed Graphical Models in High-Dimensional Data. R package version 1.2-1

Hoorelbeke, K., Marchetti, I., De Schryver, M., \& Koster, E. H. W. (2016). The interplay between cognitive risk and resilience factors in remitted depression: A network analysis. Journal of Affective Disorders, 195, 96-104. doi:10.1016/j.jad.2016.02.001

Kernis, M. H. (2003). Toward a Conceptualization of Optimal Self-Esteem. Psychological Inquiry, 14(1), 1-26. doi:10.1207/s15327965pli1401_01

Kross, E., Verduyn, P., Demiralp, E., Park, J., Lee, D. S., Lin, N., ... Ybarra, O. (2013). Facebook Use Predicts Declines in Subjective Well-Being in Young Adults. Plos One, 8(8), e69841. doi:10.1371/journal.pone.0069841

Kruis, J., \& Maris, G. (2016). Three representations of the Ising model. Scientific Reports, 6(1). doi:10.1038/srep34175

Lovibond, P. F., \& Lovibond, S. H. (1995). The structure of negative emotional states: Comparison of the Depression Anxiety Stress Scales (DASS) with the Beck Depression and Anxiety Inventories. Behaviour Research and Therapy, 33(3), 335-343. doi:10.1016/0005-7967(94)00075-u

McLaughlin, K. A., \& Nolen-Hoeksema, S. (2011). Rumination as a transdiagnostic factor in depression and anxiety. Behaviour Research and Therapy, 49(3), 186-193. doi:10.1016/j.brat.2010.12.006

Opsahl, T., Agneessens, F., \& Skvoretz, J. (2010). Node centrality in weighted networks: Generalizing degree and shortest paths. Social Networks, 32(3), 245-251. doi:10.1016/j.socnet.2010.03.006

Orth, U., Robins, R. W., Trzesniewski, K. H., Maes, J., \& Schmitt, M. (2009). Low self-esteem is a risk factor for depressive symptoms from young adulthood to old age. Journal of Abnormal Psychology, 118(3), 472-478. doi:10.1037/a0015922 
Orth, U., Robins, R. W., \& Widaman, K. F. (2012). Life-span development of self-esteem and its effects on important life outcomes. Journal of Personality and Social Psychology, 102(6), 1271-1288. doi:10.1037/a0025558

Ozimek, P., \& Bierhoff, H.-W. (2016). Facebook use depending on age: The influence of social comparisons. Computers in Human Behavior, 61, 271-279. doi:10.1016/j.chb.2016.03.034

Paradise, A. W., \& Kernis, M. H. (1999). Development of the Contingent Self-esteem Scale. Unpublished data, University of Georgia.

Pettijohn II, T. F., LaPiene, K. E., Pettijohn, T. F., \& Horting, A. L. (2012). Relationships between Facebook Intensity, Friendship Contingent Self-Esteem, and Personality in U.S. College Students. Cyberpsychology: Journal of Psychosocial Research on Cyberspace, 6(1). doi:10.5817/cp2012-1-2

Rhemtulla, M., van Bork, R., \& Borsboom, D. (2018). Worse than measurement error: Consequences of inappropriate latent variable measurement models. Preprint retrieved on January 25, 2019 from the Open Science Framework website: https://osf.io/27bxg/

Rosen, L. D., Whaling, K., Carrier, L. M., Cheever, N. A., \& Rokkum, J. (2013). The Media and Technology Usage and Attitudes Scale: An empirical investigation. Computers in Human Behavior, 29(6), 2501-2511. doi:10.1016/j.chb.2013.06.006

Rosenberg, M. (1965). Society and the adolescent self-image. Princeton, NJ: Princeton University Press.

Rutledge, C. M., Gillmor, K. L., \& Gillen, M. M. (2013). Does this profile picture make me look fat? Facebook and body image in college students. Psychology of Popular Media Culture, 2(4), 251-258. doi:10.1037/ppm0000011

Schmittmann, V. D., Cramer, A. O. J., Waldorp, L. J., Epskamp, S., Kievit, R. A., \& Borsboom, D. (2013). Deconstructing the construct: A network perspective on psychological 
phenomena. New Ideas in Psychology, 31(1), 43-53. doi:10.1016/j.newideapsych.2011.02.007

Shaw, A. M., Timpano, K. R., Tran, T. B., \& Joormann, J. (2015). Correlates of Facebook usage patterns: The relationship between passive. Facebook use, social anxiety symptoms, and brooding. Computers in Human Behavior, 48, 575-580. doi: 10.1016/j.chb.2015.02.003

Skierkowski, D., \& Wood, R. M. (2012). To text or not to text? The importance of text messaging among college-aged youth. Computers in Human Behavior, 28(2), 744-756. doi:10.1016/j.chb.2011.11.023

Sowislo, J. F., \& Orth, U. (2013). Does low self-esteem predict depression and anxiety? A metaanalysis of longitudinal studies. Psychological Bulletin, 139(1), 213-240. doi:10.1037/a0028931

Statista (2017). Daily time spent on social networking by internet users worldwide from 2012 to 2017 (in minutes). Retrieved from https://www.statista.com/statistics/433871/dailysocial-media-usage-worldwide/

Steers, M. N. (2016) 'It's complicated': Facebook's relationship with the need to belong and depression. Current Opinion in Psychology, 9, 22-26. doi: 10.1016/j.copsyc.2015.10.007

Steers, M. N., Wickham, R. E., \& Acitelli, L. K. (2014). Seeing everyone else's highlight reels: How Facebook usage is linked to depressive symptoms. Journal of Social and Clinical Psychology, 33(8), 701-731. doi:10.1521/jscp.2014.33.8.701

Stefanone, M. A., Lackaff, D., \& Rosen, D. (2011). Contingencies of Self-Worth and SocialNetworking-Site Behavior. Cyberpsychology, Behavior, and Social Networking, 14, 41-49. doi:10.1089/cyber.2010.0049 
Treynor, W., Gonzalez, R., \& Nolen-Hoeksema, S. (2003). Rumination reconsidered: A psychometric analysis. Cognitive therapy and research, 27(3), 247-259. doi:10.1023/a:1023910315561

van Borkulo, Epskamp, \& Millner (2016). NetworkComparisonTest: Statistical Comparison of Two Networks Based on Three Invariance Measures. R Package version 2.0.1

van Borkulo, C. D., Boschloo, L., Kossakowski, J. J., Tio, P., Schoevers, R. A., Borsboom, D., \& Waldorp, L J. (submitted). Comparing network structures on three aspects: A permutation test. doi: 10.13140/RG.2.2.29455.38569

Verduyn, P., Lee, D. S., Park, J., Shablack, H., Orvell, A., Bayer, J., ... Kross, E. (2015). Passive Facebook usage undermines affective well-being: Experimental and longitudinal evidence. Journal of Experimental Psychology: General, 144(2), 480-488. doi:10.1037/xge0000057

Verduyn, P., Ybarra, O., Résibois, M., Jonides, J., \& Kross, E. (2017). Do Social Network Sites Enhance or Undermine Subjective Well-Being? A Critical Review. Social Issues and Policy Review, 11(1), 274-302. doi:10.1111/sipr.12033

Vogel, E. A., Rose, J. P., Roberts, L. R., \& Eckles, K. (2014). Social media, social comparison, and self-esteem. Psychology of Popular Media Culture, 3(4), 206-222. doi:10.1037/e512142015-699

Wang, J. L., Wang, H. Z., Gaskin, J., \& Hawk, S. (2017). The Mediating Roles of Upward Social Comparison and Self-esteem and the Moderating Role of Social Comparison Orientation in the Association between Social Networking Site Usage and Subjective Well-Being. Frontiers in Psychology, 8. doi:10.3389/fpsyg.2017.00771

Wilson, R. E., \& Gosling, S. D. (2016). A review of Facebook research in the social sciences. Perspectives on Psychological Science, 7(3) 203-220 doi:10.31219/osf.io/2r92d 
Wouters, S., Duriez, B., Luyckx, K., Klimstra, T., Colpin, H., Soenens, B., \& Verschueren, K. (2013). Depressive symptoms in university freshmen: Longitudinal relations with contingent self-esteem and level of self-esteem. Journal of Research in Personality, 47(4), 356-363. doi:10.1016/j.jrp.2013.03.001

Woods, H. C., \& Scott, H. (2016). \#Sleepyteens: Social media use in adolescence is associated with poor sleep quality, anxiety, depression and low self-esteem. Journal of Adolescence, 51, 41-49. doi:10.1016/j.adolescence.2016.05.008

Zhao, S., Grasmuck, S., \& Martin, J. (2008). Identity construction on Facebook: Digital empowerment in anchored relationships. Computers in Human Behavior, 24(5), 18161836. doi:10.1016/j.chb.2008.02.012

Zhao, T., Li, X., Liu, H., Roeder, K., Lafferty, J., \& Wasserman, L. (2015). huge: HighDimensional Undirected Graph Estimation. R package version 1.2.7.

Zuo, A. (2015). Measuring up: Social comparisons on Facebook and contributions to selfesteem and mental health (Doctoral dissertation). Retrieved from Deep Blue, University of Michigan Library. 